\title{
Expression of CDCA8 correlates closely with FOXM1 in breast cancer: public microarray data analysis and immunohistochemical study
}

\author{
D. C. JIAO ${ }^{\ddagger}$ Z. D. LU*, J. H. QIAO, M. YAN, S. D. CUI, Z. Z. LIU* \\ Breast Cancer Center, Affiliated Cancer Hospital of Zhengzhou University, Henan Cancer Hospital, Zhengzhou 45008 P.R. China \\ *Correspondence: zliuoncol@163.com \\ ${ }^{*}$ Contributed equally to this work.
}

Received September 3, 2014 / Accepted November 19, 2014

\begin{abstract}
Forkhead Box M1 (FOXM1) is an oncogenic transcription factor implicated in breast cancer progression and metastasis. However, the clinical significance of FOXM1 and its associated signaling genes in human breast cancer still needed to be clarified. In this study, we first analyzed the co-expression gene pattern of FOXM1 in three breast cancer gene expression microarray datasets from the Oncomine database. Cell division cycle associated 8 (CDCA8) gene was identified to correlate closely with FOXM1. In silico analysis further indicated that CDCA8 overexpressed in breast cancer tissues compared with the normal controls is significantly associated with the triple-negative phenotype. Experimentally, we performed a immunohistochemical study to detect the expression of CDCA8 in 112 breast cancer samples, and evaluated its clinicopathological and prognostic significance. We found that CDCA8 was frequently over-expressed in breast cancer tissues, and increased expression of CDCA8 was positively associated with FOXM1 expression, triple-negative phenotype and shorter overall survival. Moreover, we also found that combination of CDCA8 and FOXM1 showed a higher hazard ratio than the individual markers. Our results suggest that FOXM1-CDCA8 signature might be involved in breast cancer progression, and serves as a potential prognostic factor and a promising therapeutical target.
\end{abstract}

Key words: breast cancer, FOXM1, CDCA8, prognosis, microarray

Breast cancer accounts for about $15 \%$ of all female cancer deaths worldwide. China's incidence of breast cancer has increased rapidly in recent years, and has now become the most common cancer in females in some major cities [1]. Breast cancer is a highly heterogeneous disease, however, the currently classification systems based on TNM stages or estrogen receptor (ER), progesterone receptor (PR), and human epidermal growth factor receptor 2 (Her2) status are not enough reliable to identify those subgroups with poor clinical outcomes. Thus, it is of utmost importance to exploring the underlying mechanisms by which breast cancer cells progress, and identify novel molecular predictors for patients with breast cancer.

FOXM1, as an oncogenic transcription factor, has been found to be overexpressed in multiple cancers including breast cancers [2]. Recent experimental studies have suggested that activation of FOXM1 and its downstream target genes contribute to aggressive tumor growth, angiogenesis and invasion, resulting in tumor progression [3]. Furthermore, combined analysis of FOXM1 and its targets also showed prognostic implication in clinical cancer samples $[4,5]$.

In this study, by in silico analysis on public microarray data and immunohistochemical study, we found CDCA8 being a novel FOXM1-related gene overexpressed in breast cancers. Moreover, aberrant FOXM1-CDCA8 expression could predict poor prognosis in a cohort of breast cancer patients. Our data suggests the FOXM1-CDCA8 pathway may play important role in breast cancer progression.

\section{Materials and methods}

Datasets. Three independent gene expression microarray datasets (Bitter Breast, Curtis Breast, TCGA Breast) deposited in the Oncomine database were used in bioinformatics analysis [6]. Bitter Breast dataset comprises 19,574 gene expression data of 336 breast tumor samples measured using Human Genome U133 Plus 2.0 Array microarray platform. Curtis Breast dataset comprises the mRNA expression data of 19,273 genes from 
2,136 breast cancer and normal breast samples measured using Illumina HumanHT-12 V3.0 R2 Array platform. TCGA (The Cancer Genome Atlas) Breast dataset comprises gene expression data of 20,423 measured genes from 593 breast samples.

Bitter Breast, Curtis Breast and TCGA Breast datasets were used to mine the co-expression pattern of FOXM1. For no normal breast samples were included in Bitter Breast dataset, only Curtis Breast and TCGA Breast datasets were used to compare the expression levels of CDCA8 in breast cancer and normal breast samples.

In silico analysis. Coexpression analysis was performed using the Oncomine database (http://oncomine.org) as previously described $[7,8]$. The threshold was set as follows: P-value $<1 \mathrm{E}-4$; fold-change $>2$ and gene rank $<$ top $1 \%$; filtered gene: FOXM1; filtered analysis type: Coexpression Analysis; Datasets Name: Bittner Breast, Curtis Breast, TCGA Breast. The top 20 coexpressed genes were extracted and overlapped. Then the top co-expressed gene (CDCA8) was filtered to validate if FOXM1 ranks among the top list of its co-expression genes.

The threshold to compare the CDCA8 expression in breast cancers and normal breasts was set as follows: Gene: CDCA8; Analysis Type: Differential Analysis - Cancer vs. Normal Analysis; Dataset Name: Dataset Name: Curtis Breast, TCGA Breast. Then the results were grouped by patients' characteristics to evaluate their association with CDCA8 expression.

Clinical samples. A total of 112 formalin-fixed paraffinembedded breast cancer specimens were retrospectively collected with informed consent between January 2005 and January 2010. Expression status of ER, PR and HER2 was also reviewed and extracted. All patients received adequate local treatment (breast conserving surgery or total mastectomy) with complete axillary dissection. Post-surgery treatments were performed according to the guideline recommendations of Chinese Anti-Cancer Association. No patient received neo-adjuvant therapy. All enrolled cases had complete data on tumor characteristics and follow-up data. The clinicopathological data of clinical samples were summarized in table 1 . The study was approved by the ethnic committees of our local institution.

Immunohistochemistry. Specimens were sectioned into $4-\mu \mathrm{m}$ thick slices, and subjected to immunostaining of CDCA8 and FOXM1 protein. Briefly, after heating antigen-retrieval in $0.1 \mathrm{M}$ sodium citrate for 10 minutes, and treated with $3 \%$ hydrogen peroxide for $15 \mathrm{~min}$, sections were incubated with primary antibodies (Santa Cruz, 1:100 dilution), and then visualized using the avidin-biotin-peroxidase complex method. The level of CDCA8 and FOXM1 expression was semi-quantified using $\mathrm{H}$-score analysis as previously [9]. $\mathrm{H}$ scores were calculated by multiplying the percentage (1: $<10 \% ; 2: 10 \%-30 \% ; 3:>30 \%)$ and staining intensity (0: none or weak staining; 1 : medium staining; 2 : strong staining). The median scores were used as cutoff values to classify breast cancer samples into high and low expression subgroups for each biomarker.
Statistics. For immunohistochemical results, the association between CDCA8 expression levels with clicopathological features was determined with $\chi^{2}$ test. The Kaplan-Meier method was used for survival analysis in terms of single or combined biomarkers and evaluated by the log-rank test. Multivariate survival analyses were performed using the Cox proportional hazards model. All statistics were performed using MedCalc 11.4 software, and $\mathrm{P}<0.05$ was considered statistically significant.

\section{Results}

Using the Oncomine integrated cancer profiling database, FOXM1 were searched for coexpressed genes in the three

Table 1. Association of CDCA8 expression with patients' clinicopathologic features in breast carcinomas.

\begin{tabular}{|c|c|c|c|c|}
\hline \multirow[b]{2}{*}{ Variable } & \multirow[b]{2}{*}{ All Cases } & \multicolumn{2}{|c|}{ CDCA8 } & \multirow[b]{2}{*}{$P$ value } \\
\hline & & $\begin{array}{c}\text { Low } \\
\text { Expression }\end{array}$ & $\begin{array}{c}\text { High } \\
\text { Expression }\end{array}$ & \\
\hline \multicolumn{5}{|c|}{ Age (years)* } \\
\hline$<52.8$ & 66 & 38 & 28 & 0.0839 \\
\hline$\geq 52.8$ & 46 & 18 & 28 & \\
\hline \multicolumn{5}{|l|}{ Grade } \\
\hline 1 & 6 & 2 & 4 & 0.3655 \\
\hline 2 & 101 & 51 & 50 & \\
\hline 3 & 5 & 3 & 2 & \\
\hline \multicolumn{5}{|l|}{ pT status } \\
\hline $\mathrm{T} 1$ & 22 & 9 & 13 & 0.3762 \\
\hline $\mathrm{T} 2$ & 79 & 41 & 38 & \\
\hline $\mathrm{T} 3$ & 11 & 6 & 5 & \\
\hline \multicolumn{5}{|l|}{ pN status } \\
\hline- & 76 & 40 & 36 & 0.057 \\
\hline+ & 36 & 16 & 20 & \\
\hline \multicolumn{5}{|c|}{ TNM stage } \\
\hline I & 6 & 2 & 4 & 0.3608 \\
\hline II & 67 & 37 & 30 & \\
\hline III & 39 & 17 & 22 & \\
\hline \multicolumn{5}{|l|}{ ER } \\
\hline- & 43 & 17 & 26 & 0.1201 \\
\hline+ & 69 & 39 & 30 & \\
\hline \multicolumn{5}{|l|}{ PR } \\
\hline- & 50 & 23 & 27 & 0.5685 \\
\hline+ & 62 & 33 & 29 & \\
\hline \multicolumn{5}{|l|}{ HER2 } \\
\hline- & 81 & 41 & 40 & 1.0000 \\
\hline+ & 31 & 15 & 16 & \\
\hline \multicolumn{5}{|c|}{ Triple-negative } \\
\hline No & 87 & 49 & 38 & 0.0233 \\
\hline Yes & 25 & 7 & 18 & \\
\hline \multicolumn{5}{|c|}{ FOXM1 expression } \\
\hline Low & 50 & 39 & 11 & $<0.001$ \\
\hline High & 62 & 17 & 45 & \\
\hline
\end{tabular}

*: Mean age 
largest breast cancer datasets. Top 10 co-expressed genes of FOXM1 from each dataset were compared, and MELK and CDCA 8 were overlapped in all the three datasets (Supplemental data Figure S). MELK is a known FOXM1-related gene as a mediator for its phosphorylation and activation[10]. The association between CDCA8 with FOXM1 has not been identified. Curtis Breast and TCGA Breast datasets were further used to determine the expression pattern of CDCA8 in breast cancers. As seen in Figure 1, all of the two datasets showed a significantly higher level of CDCA8 in breast cancer compared with normal breast tissues (Figure 1A, 1B). In silico study further indicated that triple-negative breast cancer group had a higher CDCA8 levels than the other groups in both datasets (Figure 1C, 1D), and there is also a trend that the expression of CDCA8 increased with the tumor grade (Figure 1E).

A

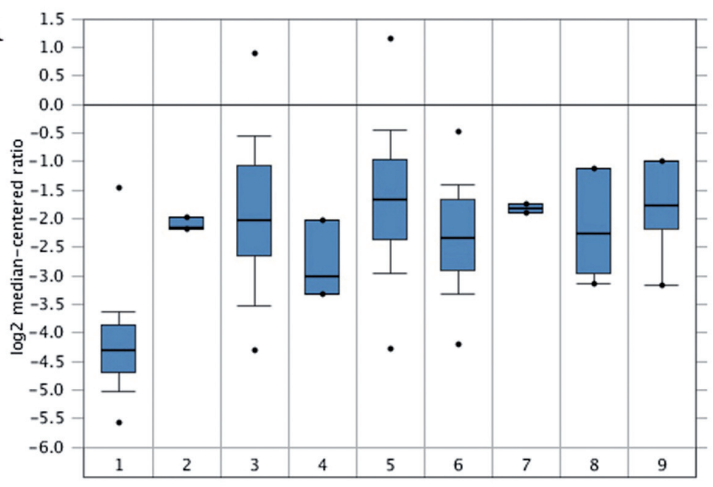

To validate this observation, immunohistochemistry was then performed to evaluate the expression of CDCA8 in a cohort of surgically resected breast cancer samples. FOXM1 positive staining was mainly located in the nucleus of cancer cells in breast cancer tissues (Figure 2A, 2B), while CDCA 8 demonstrated nuclear and cytoplasmic staining pattern (Figure 2C, 2D). No or weak staining was observed in normal breast tissues. Our results indicated that the expression levels of FOXM1 correlated closely with CDCA8 expression in this cohort, with high FOXM1 expression group containing more patients with high CDCA8 expression than low FOXM1 group $(72.6 \%$ vs. $22.0 \%, \mathrm{P}<0.001)$ (Table 1).

The association between expression of CDCA8 with the common characteristics and well known clinicopathological prognostic variables was also evaluated. As seen Table 1, no

B

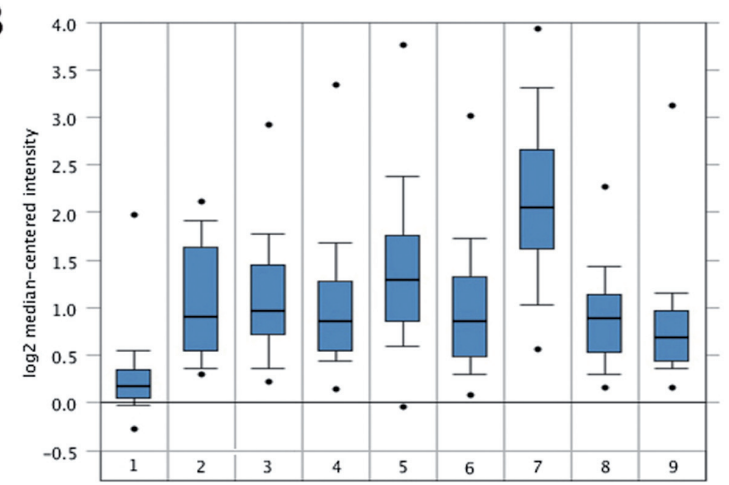

$\mathrm{C}$

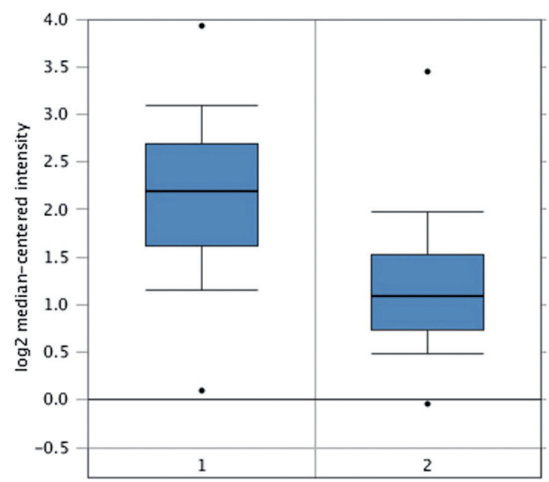

$\mathrm{D}$

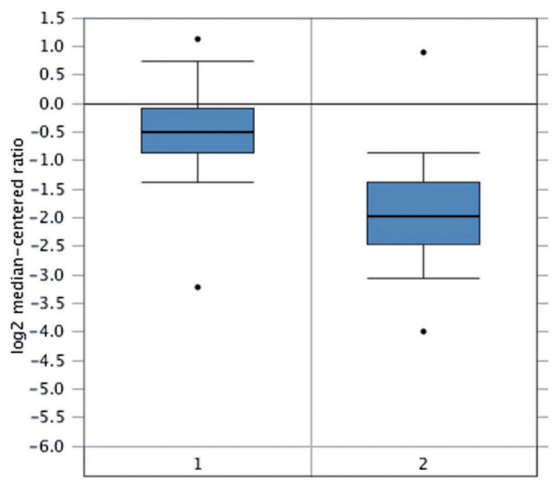

$\mathrm{E}$

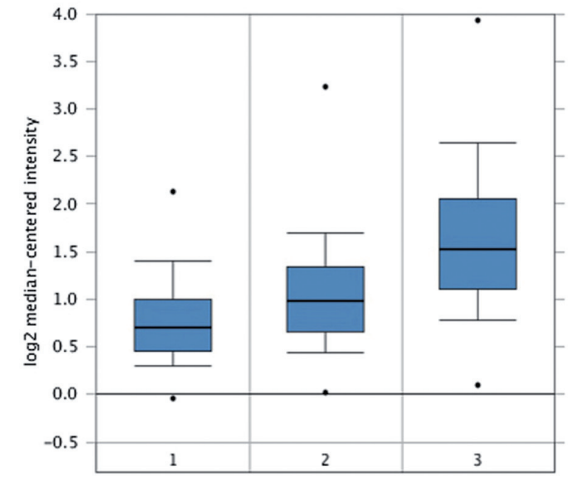

Figure 1. In silico analysis on CDCA8 expression pattern in Oncomine database. A. CDCA8 in normal breast tissues and different types of breast cancers in Curtis dataset. 1: Breast (61); 2: Intraductal Cribriform Breast Adenocarcinoma (3); 3: Invasive Breast Carcinoma (76); 4: Invasive Ductal and labular carcinoma (3); 5: Invasive Ductal Breast Carcinoma (389); 6: Invasive Lobular Breast Carcinoma (36); 7: Male Breast Carcinoma (3); 8: Mixed Lobular and Ductal Breast Carcinoms (7); 9: Mucinous Breast Carcinoma (4). B. CDCA8 in normal breast tissues and different types of breast cancers inTCGA Breast dataset. 1: Breast (144); 2: Breast caricinoma (14); 3: Invasive Breast carcinoma (21); 4: Invasive Ductal and Invasive Lobular Breast Carcinoma (90); 5: Invasive Ductal Breast Carcinoma (1556); 6: Invasive Lobular Breast Carcinoma (148); 7: Medullary Breast Carcinoms (32); 8: Mucinous Breast Carcinoma (46); 9: Tubular Breast Carcinoma (67). C. CDCA8 expression in triple negative breast cancer compared with other types in TCGA dataset. 1: Triple-negative (250); 2: Other Biomarker Status (1725). D. CDCA8 expression in triple negative breast cancer compared with other types in Curtis Breast dataset. 1: Triple-negative (49); 2: Other Biomarker Status (300). E. CDCA8 expression in different grade subgroups in Curtis Breast dataset. 1: Grade 1 (170); 2: Grade 2 (775); 3: Grade 3 (957). 

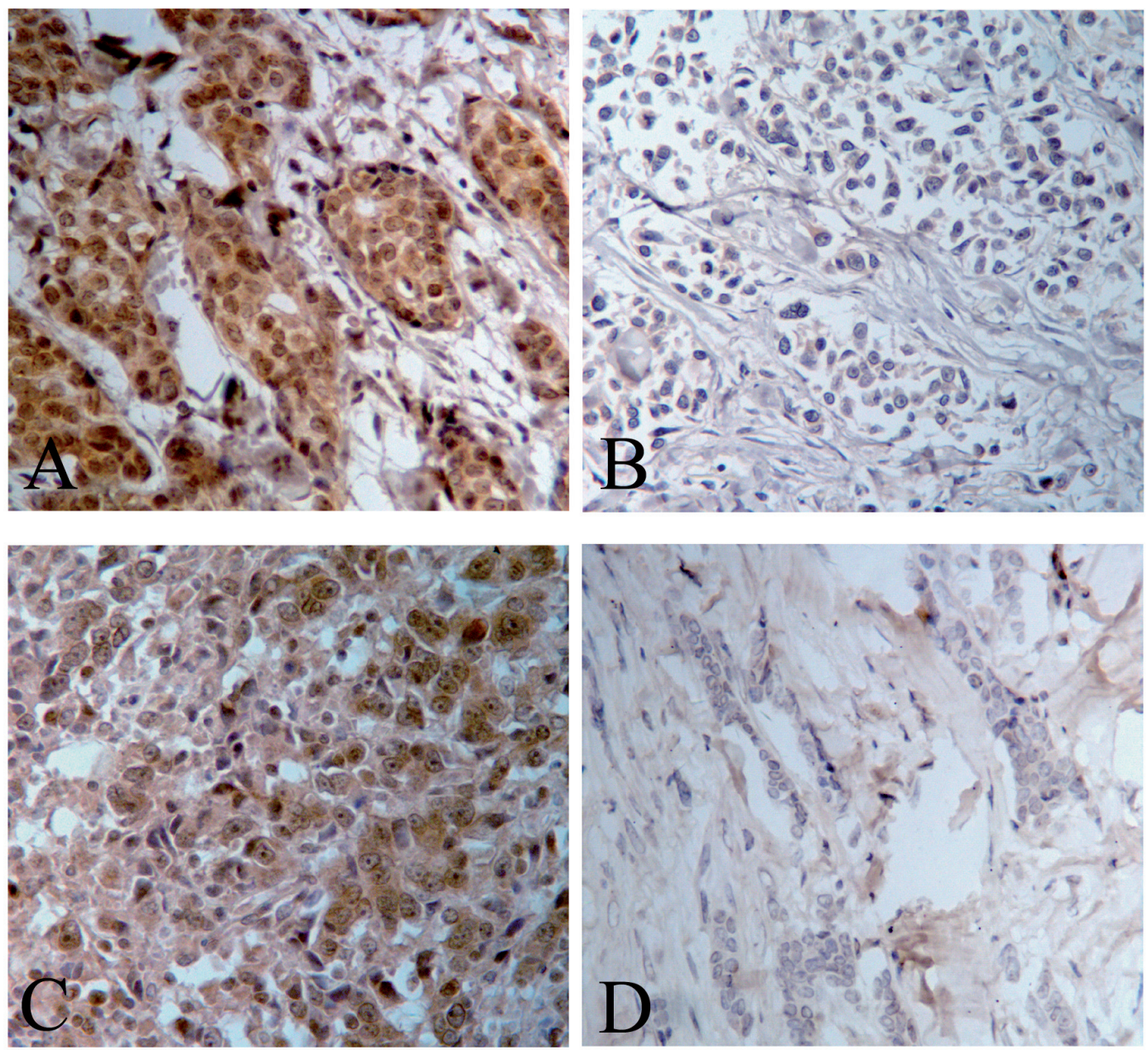

Figure 2. Representative images of immunohistochemistry. A: High FOXM1 staining in breast cancer tissues; B: Low FOXM1 staining in breast cancer tissues; C: CDCA8 staining in breast cancer tissues; D: Low CDCA8 staining in breast cancer tissues.

association was found between CDCA8 expression and clinicopathological characteristics except for the triple-negative phenotype ( $47.3 \%$ vs. $12.5 \%, \mathrm{p}=0.0233)$. And there was also a significant association between FOXM1 expression with triple-negative phenotype $(\mathrm{P}=0.0334)$.

Assessment of patients' survival revealed that increased expression of CDCA 8 and FOXM1 each was closely associated with poor disease-specific survival (Figure 3A, 3B). In multivariate analyses, only FOXM1 but not CDCA8 was identified as an independent prognostic factor for overall survival, along with HER2 status and triple negative phenotype (Table 2). Furthermore, when the combined pattern of FOXM1 and CDCA8 expression was analyzed, we further found that the subgroup with high expression of both markers had the worst survival (Figure 3C). And combined FOXM1 and CDCA8 expression demonstrated a higher hazard ratio (3.7443, 95\% CI range: 1.8872-7.4290) than the single markers (Table 2).

\section{Discussion}

The oncogenic transcription factor Forkhead Box M1 (FOXM1) is specifically overexpressed in a majority of human carcinomas, but is usually low in normal cells, which make it to be an attractive target for anticancer drugs $[11,12]$. However, the exact roles of FOXM1 pathway during cancer progression are still needed to be clarified. The identification of specific coexpressed genes of FOXM1 is not only helpful to better understand its molecular mechanisms as an oncogene, but also to explore its clinical significance, especially in subgroup classification and prognosis prediction for cancer patients.

In this present study, for the first time, we identified a close association between FOXM1 with a novel chromosomal passenger complex (CRC) component CDCA8, at least in breast carcinomas. We discovered several lines of evidence supporting that CDCA8 might be a new FOXM1 related genes. First, CDCA 8 ranks the top of co-expressed genes of FOXM1 

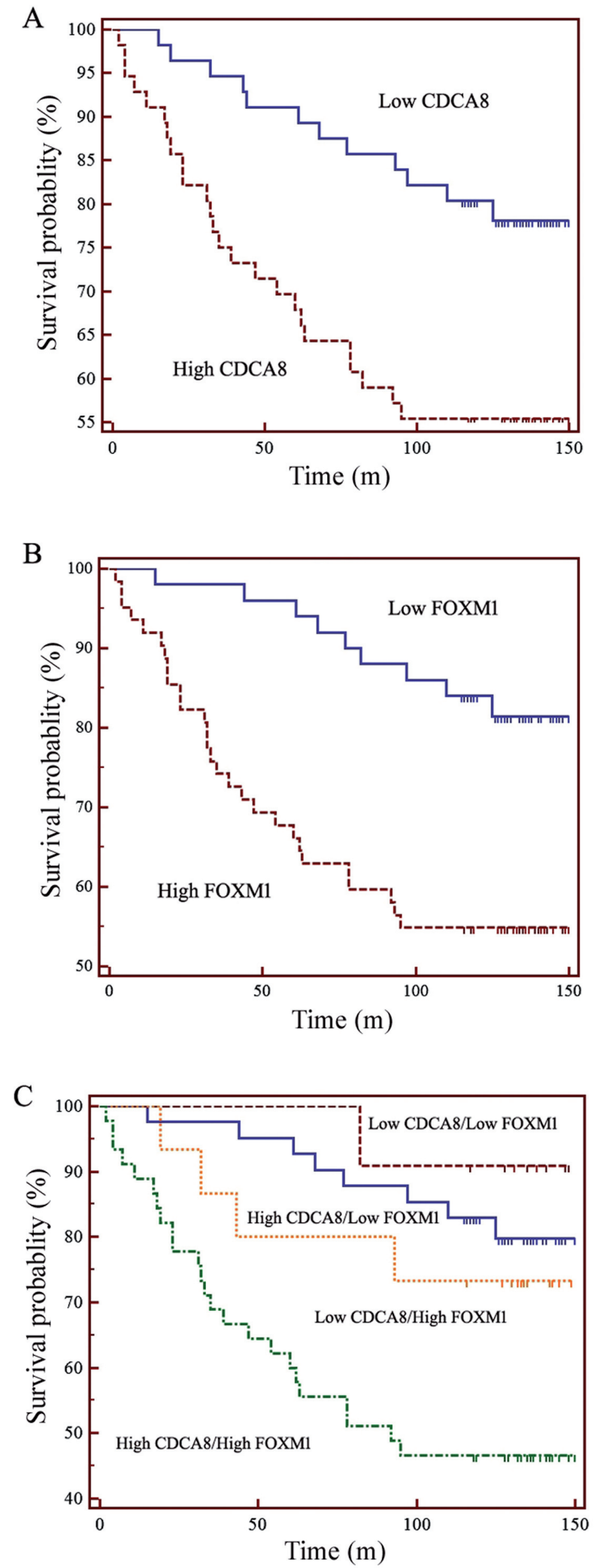

Figure 3. Kaplan-Meier survival analysis for expression of single and combined biomarkers. A: CDCA8; B: FOXM1; C: combined CDCA8 and FOXM1. in three largest gene expression microarray dataset of breast cancer. Secondly, both our in silico analysis and immunohistochemical results revealed that like FOXM1, the expression of CDCA8 is also specifically upregulated in breast cancer tissues. Third, the level of CDCA8 expression was further validated to be correlated to FOXM1 in clinical breast cancer samples by immunohistochemistry.

As a potential oncogene, FOXM1 is activated by oncogenic Ras-MAPK, Sonic Hedgehog, NF-kB and EGFR pathways, and negatively regulated by tumor suppressor p53 [13-17]. When FOXM1 becomes overactivated in cancers, it abberently induce cycle progression by transcriptionally activate genes that are involved in cell cycle such as Cyclin B, Cdc25b phosphatase, Plk1, Skp2 and Cks1[18]. Chromosomal passenger complex comprises CDCA8, survivin, Aurora B kinase, and INCENP [19]. Interestingly, survivin and Aurora B kinase has been found to be transcriptionally activated by FOXM1 [18]. Therefore, the close association between FOXM1 and CDCA8 at gene expression levels identified in this study supports that CDCA8 could be a downstream effective factor of FOXM1. Furthermore, our and several other findings suggest also that FOXM1-chromosomal passenger complex pathway might play a crucial role linking cell division to cancer, and inhibition of this pathway could be a candidate for treatment strategy of breast cancer.

In this study, for the first time the clinical significance of CDCA8 in breast cancer has been reported. We found that CDCA8 correlated with a more progressive phenotype, the triple-negative breast cancer. Although associated with poor prognosis of breast cancer patients, CDCA8 was not an independent prognostic factor. However, combination of FOXM1 and CDCA8 showed more reliable prognostic value than the single markers. Thus, we speculated that CDCA8 expression may influence the FOXM1-related prognosis in breast carcinoma, and that FOXM1-CDCA8 signaling may play a more significant role in the genesis and progression of this malignancy.

In summary, this study provided in silico and clinical evidence supporting that CDCA8 is a new FOXM1 associated gene, and we also found that combination of both markers would be a promising prognostic factor for breast cancer patients. These findings suggest that aberrant FOXM1-CDCA8 signaling might represent a malignant feature of breast cancer cells. The exact mechanism deserves further investigation.

\section{References}

[1] LI J, ZHANG B N, FAN J H, PANG Y, ZHANG P et al. A nation-wide multicenter 10-year (1999-2008) retrospective clinical epidemiological study of female breast cancer in China. BMC Cancer 2011; 11: 364. http://dx.doi. org/10.1186/1471-2407-11-364

[2] HALASI M, GARTEL A L. Targeting FOXM1 in cancer. Biochem Pharmacol 2013; 85: 644-652. http://dx.doi. org/10.1016/j.bcp.2012.10.013 
[3] BELLA L, ZONA S, NESTAL DE MORAES G, LAM E W. FOXM1: A key oncofoetal transcription factor in health and disease. Semin Cancer Biol 2014. http://dx.doi.org/10.1016/i. semcancer.2014.07.008

[4] LI D, WEI P, PENG Z, HUANG C, TANG $\mathrm{H}$ et al. The critical role of dysregulated FOXM1-PLAUR signaling in human colon cancer progression and metastasis. Clin Cancer Res 2013; 19: 62-72. http://dx.doi.org/10.1158/1078-0432.CCR12-1588

[5] KIM S K, ROH Y G, PARK K, KANG T H, KIM W J et al. Expression signature defined by FOXM1-CCNB1 activation predicts disease recurrence in non-muscle-invasive bladder cancer. Clin Cancer Res 2014; 20: 3233-3243. http://dx.doi. org/10.1158/1078-0432.CCR-13-2761

[6] RHODES D R, KALYANA-SUNDARAM S, MAHAVISNO V, VARAMBALLY R, YU J et al. Oncomine 3.0: genes, pathways, and networks in a collection of 18,000 cancer gene expression profiles. Neoplasia 2007; 9: 166-180. http://dx.doi. org/10.1593/neo.07112

[7] VARISLI L. Meta-analysis of the expression of the mitosisrelated gene Fam83D. Oncol Lett 2012; 4: 1335-1340.

[8] WILSON B J, GIGUERE V. Meta-analysis of human cancer microarrays reveals GATA3 is integral to the estrogen receptor alpha pathway. Mol Cancer 2008; 7: 49. http://dx.doi. org/10.1186/1476-4598-7-49

[9] THIKE A A, CHNG M J, FOOK-CHONG S, TAN P H. Immunohistochemical expression of hormone receptors in invasive breast carcinoma: correlation of results of $\mathrm{H}$-score with pathological parameters. Pathology 2001; 33: 21-25.

[10] JOSHI K, BANASAVADI-SIDDEGOWDA Y, MO X, KIM S $\mathrm{H}, \mathrm{MAO} \mathrm{P}$ et al. MELK-dependent FOXM1 phosphorylation is essential for proliferation of glioma stem cells. Stem Cells 2013; 31: 1051-1063. http://dx.doi.org/10.1002/stem.1358

[11] GARTEL A L. The oncogenic transcription factor FOXM1 and anticancer therapy. Cell Cycle 2012; 11: 3341-3342. http:// dx.doi.org/10.4161/cc.21841
[12] GARTEL A L. FoxM1 inhibitors as potential anticancer drugs. Expert Opin Ther Targets 2008; 12: 663-665. http://dx.doi. org/10.1517/14728222.12.6.663

[13] MAJOR M L, LEPE R, COSTA R H. Forkhead box M1B transcriptional activity requires binding of Cdk-cyclin complexes for phosphorylation-dependent recruitment of p300/ CBP coactivators. Mol Cell Biol 2004; 24: 2649-2661. http:// dx.doi.org/10.1128/MCB.24.7.2649-2661.2004

[14] TEH M T, WONG S T, NEILL G W, GHALI L R, PHILPOTT M P ET AL. FOXM1 is a downstream target of Gli1 in basal cell carcinomas. Cancer Res 2002; 62: 4773-4780.

[15] PENZO M, MASSA P E, OLIVOTTO E, BIANCHI F, BORZI $\mathrm{R} \mathrm{M}$ et al. Sustained NF-kappaB activation produces a shortterm cell proliferation block in conjunction with repressing effectors of cell cycle progression controlled by E2F or FoxM1. J Cell Physiol 2009; 218: 215-227. http://dx.doi.org/10.1002/ jcp. 21596

[16] BEKTAS N, HAAF A, VEECK J, WILD P J, LUSCHERFIRZLAFF J et al. Tight correlation between expression of the Forkhead transcription factor FOXM1 and HER2 in human breast cancer. BMC Cancer 2008; 8: 42. http://dx.doi. org/10.1186/1471-2407-8-42

[17] PANDIT B, HALASI M, GARTEL A L. p53 negatively regulates expression of FoxM1. Cell Cycle 2009; 8: 3425-3427. http://dx.doi.org/10.4161/cc.8.20.9628

[18] WANG I C, CHEN Y J, HUGHES D, PETROVIC V, MAJOR $\mathrm{M} L$ et al. Forkhead box M1 regulates the transcriptional network of genes essential for mitotic progression and genes encoding the SCF (Skp2-Cks1) ubiquitin ligase. Mol Cell Biol 2005; 25: 10875-10894. http://dx.doi.org/10.1128/ MCB.25.24.10875-10894.2005

[19] CARMENA M, WHEELOCK M, FUNABIKI H, EARNSHAW W C. The chromosomal passenger complex (CPC): from easy rider to the godfather of mitosis. Nat Rev Mol Cell Biol 2012; 13: 789-803. http://dx.doi.org/10.1038/ nrm3474 


\section{Supplementary Information}

\section{Expression of CDCA8 correlates closely with FOXM1 in breast cancer: public microarray data analysis and immunohistochemical study}

D. C. JIAO ${ }^{\ddagger}$, Z. D. LU, J. H. QIAO, M. YAN, S. D. CUI, Z. Z. LIU*

Breast Cancer Center, Affiliated Cancer Hospital of Zhengzhou University, Henan Cancer Hospital, Zhengzhou 45008 P.R. China

*Correspondence: zliuoncol@163.com

${ }^{*}$ Contributed equally to this work.

Supplementary Figure

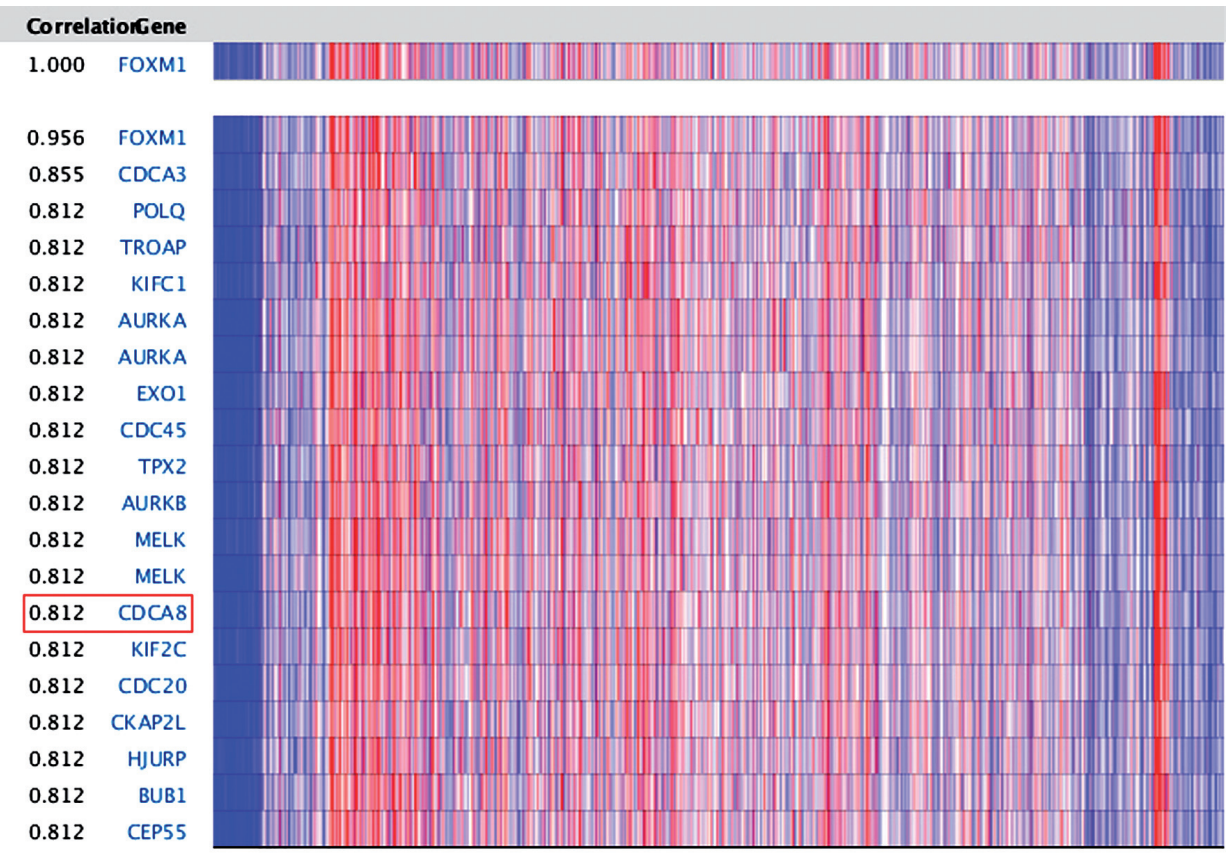



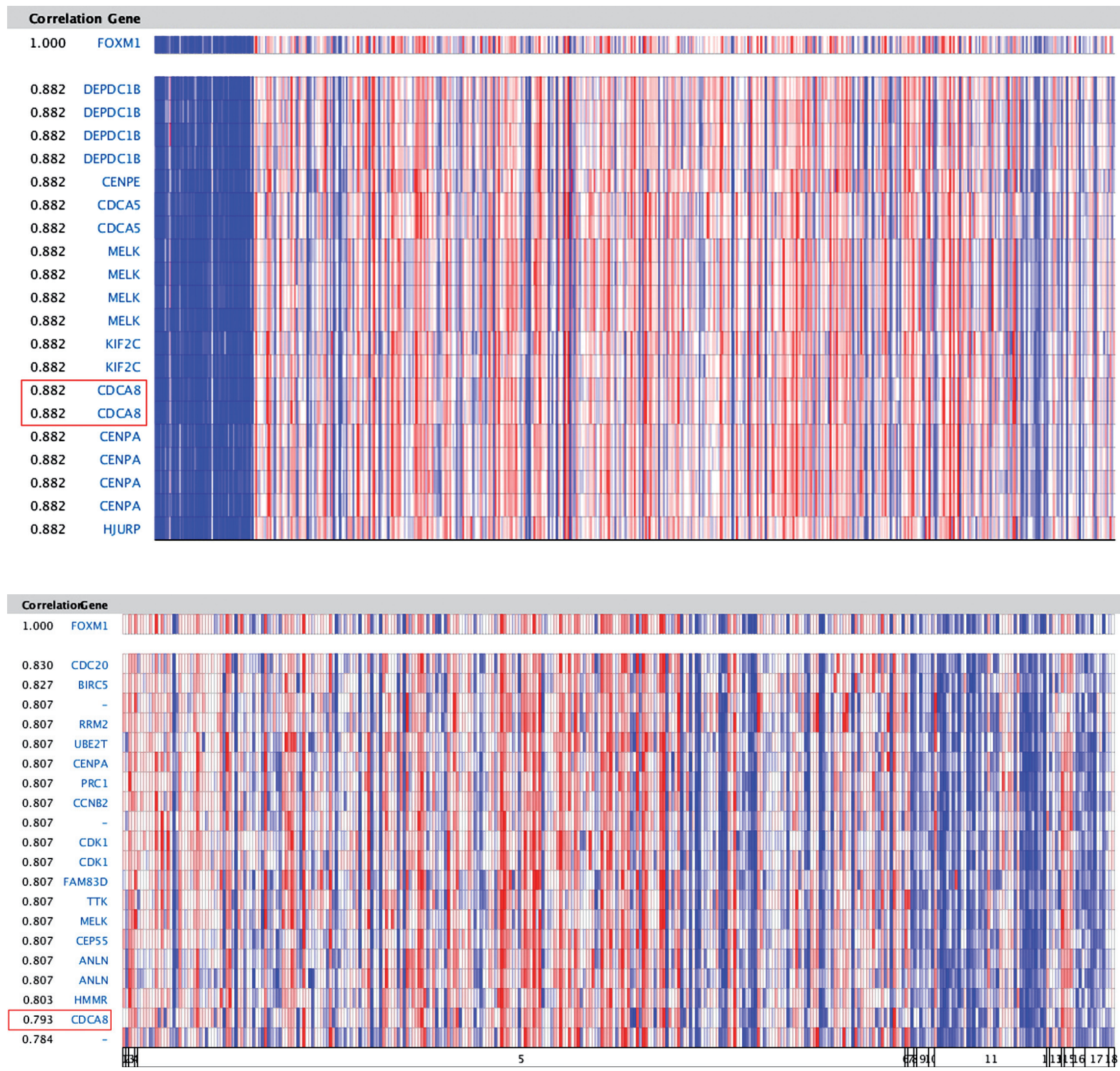

Figure S. Coexpression genes of FOXM1 in Bitter Breast, Curtis Breast, TCGA Breast datasets deposited in the Oncomine database. 